[0212-7199 (2005) 22: 12; pp 597-600] ANALES DE MEDICINA INTERNA Copyright $@ 2005$ ARAN EDICIONES, S.L.

AN. MED. INTERnA (Madrid) Vol. 22, N. ${ }^{\circ} 12$, pp. 597-600, 2005

\title{
Linfoma T/NK extraganglionar tipo nasal: caso clínico y revisión de la literatura
}

\author{
P. KHOSRAVI SHAHI, V. M. DÍAZ MUÑOZ DE LA ESPADA
}

Servicio de Oncología Médica. Hospital General Universitario Gregorio Marañón. Madrid

EXTRANODAL T/NK-CELL LYMPHOMA, NASAL TYPE: A CASE REPORT AND REVIEW OF THE LITERATURE

RESUMEN

El linfoma T/NK extraganglionar tipo nasal es un linfoma extraganglionar, que habitualmente expresa el fenotipo NK y VEB positivo, con un amplio pleomorfimo, frecuente necrosis y angioinvasión, que afecta de manera preferente a las estructuras mediofaciales, pero que también afecta a otras regiones extraganglionares. Presentamos el caso clínico de un varón de 42 años, con una masa intranasal, adenopatías submandibulares izquierdas y trombocitosis asintomática. La biopsia de la lesión intranasal consistía en un infiltrado difuso de células neoplásicas de estirpe linfoide de talla intermedia, con núcleos discretamente irregulares y pleomorfismo moderado. El inmunofenotipo de las células neoplásicas determinadas por inmunohistoquímica era: CD45+/cCD3+/Granzime B +/CD56+, CD20-/CD4/CD8-/LMP1(VEB)-. Todo ello era compatible con el diagnóstico de Linfoma NK extraganglionar tipo nasal. El estudio de extensión no mostró enfermedad a distancia. Con el diagnóstico de linfoma NK extraganglionar tipo nasal en estadio localizado con trombocitosis reactiva, el paciente inició de inmediato el tratamiento con poliquimioterapia seguido de radioterapia secuencial, decidiéndose realizar un trasplante autólogo de médula ósea en primera línea de tratamiento.

PALABRAS CLAVE: Linfoma T/NK extraganglionar. CD56. cCD3. VEB. Tipo nasal.
ABSTRACT

Extranodal T/NK-cell lymphoma, nasal type: a case report and review of the literature.

Extranodal NK/T-cell lymphoma, nasal type, is an extranodal lymphoma, usually with an NK-cell phenotype and EBV possitive, with a broad morphologic spectrum, frequent necrosis and angioinvasion, and most commonly presenting in the midfacial region, but also in other extranodal sites. We present the case of a 42-years old-man with a intranasal mass, left cervical adenopathies and indolent thrombocytosis. The biopsy of the nasal mass showed a diffuse infiltrate of medium-sized lymphoid cells, with slightly irregular nuclei and moderate polymorphous. The immunophenotype of tumor cells was CD45+, cCD3+, Granzyme B+, CD56+, CD20-,CD4-,CD8-, LMP1(EBV)-. The diagnosis of the case was Extranodal T/NK-cell lymphoma, nasal type. There was not disseminated disease in the extent studies. With the diagnosis of localized extranodal T/NK-cell lymphoma and reactive thrombocytosis, the patient was treated with chemotherapy and sequential radiotherapy, followed by bone marrow transplantation.

KEY WORDS: T/NK lymphoma extranodal. CD56. cCD3. EBV. Nasal type.

Khosravi Shahi P, Díaz Muñoz de la Espada VM. Linfoma T/NK extraganglionar tipo nasal: caso clínico y revisión de la literatura. An Med Interna (Madrid) 2005; 22: 597-600.

\section{INTRODUCCIÓN}

El linfoma T/NK extraganglionar tipo nasal es un linfoma no Hodgkin infrecuente en Europa y Estados Unidos, pero que se da con cierta frecuencia en Asia y Perú, denominado antiguamente como linfoma angiocéntrico/angioinvasivo $(1,3)$. Es una enfermedad de afectación predominantemente extragan- glionar, de curso clínico agresivo, con una pobre respuesta al tratamiento (4). Su etiología es desconocida, pero la presencia del virus Epstein-Barr es una constante, lo que sugiere un papel etiopatogénico importante en el desarrollo de esta enfermedad $(5,6)$. Deriva en su mayoría de células NK (CD2+/CD56+), y en una minoría de los casos de células $\mathrm{T}$ periféricas (CD2+/CD56-) de fenotipo T citotóxico (postímicas).

Trabajo aceptado: 26 de julio de 2005

Correspondencia: Parham Khosravi Shahi. Servicio de Oncología Médica. Hospital General Universitario Gregorio Marañón. Doctor Esquerdo, 46. 28007 Madrid. e-mail: drkhosravi@hotmail.com 
Suele afectar a estructuras extraganglionares, fundamentalmente el tracto aerodigestivo superior (fosas nasales, nasofaringe, orofaringe, cavidad oral, paladar, senos paranasales) (7). Otras localizaciones son el tracto gastrointestinal, la piel y los testículos. Las adenopatías secundarias no son ni características, ni comunes, aunque pueden estar presentes. Es excepcional que afecte a la médula ósea, y que presente expresión en sangre periférica.

Suele caracterizarse por un curso clínico rápidamente progresivo, con afectación de varias regiones extraganglionares, presentándose en estadios diseminados en el momento diagnóstico en la mayoría de los casos. Aunque puede presentarse en estadios localizados en el momento diagnóstico.

Esta enfermedad es más frecuente en el sexo masculino, y suele darse en adultos jóvenes. Su forma de presentación más común es la afectación mediofacial (masa en fosa nasal con clínica de obstrucción nasal), lo que le confiere su denominación (tipo nasal). Se trata de un linfoma de alto grado, con un pronóstico infausto (8).

\section{CASO APORTADO}

Presentamos el caso clínico de un varón de 42 años, sin alergias medicamentosas, que presenta como antecedentes personales: mononucleosis infecciosa en la infancia, fractura del brazo izquierdo hace 5 años, obesidad mórbida con esteatosis hepática tratado mediante cirugía bariatrica.

Acude a las consultas de Otorrolaringología por un cuadro de obstrucción nasal izquierda de unas semanas de evolución, con episodios repetidos de rinorrea purulenta, con sensación de taponamiento nasal izquierdo, que se acompaña de una masa intranasal y tumoración submandibular izquierda. De manera simultánea al paciente se le envía a las consultas de Hematología por una trombocitosis repetida asintomática en las analíticas. Es visto en las consultas de hematología y con la sospecha de trombocitemia esencial se realiza un aspirado/biopsia de médula ósea, presentando en el hemograma una cifra de plaquetas de 487.000/ul.

El paciente es sometido a una biopsia de la masa intranasal, que es completado con una biopsia incisional de la tumoración submandibular izquierda. La tomografía computerizada cervico-facial evidenciaba una masa de partes balndas en la celda submaxilar izquierda, con áreas de necrosis o abscesificación, de 3 centímetros de diámetro mayor; acompañado de un componente adenopático locorregional adyacente de probable naturaleza inflamatoria. Además existía una segunda lesión en discontinuidad con la anterior, correspondiente a un quiste paralateronasal izquierdo.

La biopsia de la lesión intranasal consistía en un infiltrado de células neoplásicas de estirpe linfoide con un patrón de crecimiento difuso, sin imágenes "en cielo estrellado", y con una tasa de mitosis moderada. Las células neoplásicas son de talla intermedia, con núcleos discretamente irregulares y pleomorfismo moderado. El inmunofenotipo de las células neoplásicas determinadas por inmunohistoquímica era: CD45+/cCD3+/Granzime B +/CD56+, CD20-/CD4-/CD8-/LMP1(VEB)-/Perforina-. La biopsia de la tumoración submandibular evidenciaba una infiltración masiva por células neoplásicas de estirpe linfoide de talla intermedia o grande, con irregularidades nucleares y una granulación azurofila tosca, irregular y escasa. Presentando un inmunofenotipo idéntico a la lesión intranasal. La determinación del EBER(VEB) fue positivo, había un reodenamiento policlonal de los genes de la cadena gamma del receptor del linfocito T (TCR) y de los genes de la cadena pesada de las inmunoglobulinas (IgH). Todo ello era compatible con el diagnóstico de Linfoma NK extraganglionar tipo nasal.
El paciente fue enviado de inmediato al Servicio de Oncología Médica para valoración. El paciente presentaba muy buen estado general con un performance status de 0 , sin sintomatología B y asintomático, salvo la clínica propia de la obstrucción nasal izquierda. Se completó el estudio de extensión con tomografía computerizada toraco-abdominal, que no evidenciaba diseminación a distancia, biopsia de médula ósea ya solicitada por sospecha de trombocitemia esencial, bioquímica con perfil renal, hepático, proteinograma, LDH y beta-2-microglobulina séricas.

El examen de la médula ósea mostraba un parénquima normocelular (3/5), con moderada hiperplasia megacariocítica con elementos en todos los estadios madurativos, algunos de ellos de aspecto hipertrófico, con las series restantes preservadas. No había datos de mielofibrosis, ni parásitos, ni granulomas. Tampoco había infiltración linfomatosa. El estudio de médula ósea no apoya (ni permite descartar de forma absoluta) el diagnóstico de trombocitemia esencial.

La LDH y la beta-2-microglobulina, así como el resto de los parámetros bioquímicos se mantenían en el rango de la normalidad. La serología para VIH, VHB y VHC eran negativos. Las cifras de hemoglobina y de leucocitos eran normales, con fórmula leucocitaria sin alteraciones, sólo destacaba una trombocitosis de 487.000/ul.

Con el diagnóstico de Linfoma NK extraganglionar tipo nasal en estadio localizado con trombocitosis reactiva, el paciente inició de inmediato el tratamiento con poliquimioterapia seguido de radioterapia secuencial, decidiéndose realizar un trasplante autólogo de médula ósea en primera línea de tratamiento.

\section{DISCUSIÓN}

El linfoma T/NK extraganglionar se suele dar en varones adultos jóvenes. La localización más frecuente de afectación es el trato aerodigestivo superior, siendo las cavidades nasales las estructuras afectadas de manera habitual, en forma de masa intranasal, con obstrucción nasal generalmente unilateral (sensación de taponamiento nasal, epistaxis, rinorrea purulenta y fétida, voz nasal), como en nuestro caso. Puede afectar mediante la extensión por contigüidad a las estructuras vecinas, como el paladar, los senos paranasales, las órbitas, la nasofaringe y orofaringe; lo que da lugar a exoftalmos, anisocoria con midriasis unilateral arreactiva y parálisis oculomotor. En ocasiones, puede producir extensas lesiones destructivas a nivel mediofacial, denominándose en ese caso granuloma letal de línea de media.

La afectación del tracto gastrointestinal se suele dar en forma de úlceras extensas en la mucosa gástrica, de intestino delgado y de colon, con clínica de hemorragia digestiva o perforación gastrointestinal. La afectación cutánea se presenta en forma de nódulos, muchas veces ulcerados. Puede haber síntomas $\mathrm{B}$, y en algunos casos puede acompañarse de síndrome hemofagocítico.

El diagnóstico de esta enfermedad viene dado por el estudio anatomopatológico, que muestra un infiltrado difuso de células neoplásicas linfoides, con patrón angiocéntrico, angioinvasivo y angiodestructivo, con frecuentes figuras de mitosis, y una marcada apoptosis y necrosis coagulativa, debido a la invasión y oclusión de los vasos por las células neoplásicas. Las células neoplásicas puede presentar una morfología muy variada (células de talla pequeña, mediana, grande o de aspecto anaplásico). Aunque lo más común es que esté formado por células medianas o una mezcla de células pequeñas y grandes. Dichas células presentan un núcleo irregular, y con 
nucleolo pequeño o inexistente. Acompañando a dichas células, suele haber un número variable de células inflamatorias reactivas, como linfocitos $\mathrm{B}$ y $\mathrm{T}$, histiocitos, células plasmáticas y eosinófilos.

El inmunofenotipo de las células neoplásicas determinadas por técnica de inmunohistoquímica revela el siguiente patrón: CD2+/ cCD3+(intracitoplasmático)/ CD56+ (Tabla I). Mientras que otros marcadores como CD3, CD4, CD5, CD8, CD19, CD20, CD22, CD79a resultan negativos (11) . Dos hechos característicos que ayudan al diagnóstico final es la presencia del genoma del VEB, y las moléculas o proteínas asociadas a los gránulos citotóxicos (perforina, TIA1, Granzime B) (12) . En los casos en los que las células neoplásicas presentan el inmunofenotipo de células $\mathrm{NK}(\mathrm{CD} 2+$ y CD56+) existe un reordenamiento policlonal del gen de la cadena Gamma del TCR, como en nuestro caso. Sin embargo, en ocasiones las células neoplásicas son CD2+/cCD3+/CD56-/VEB+; en estos casos las células neoplásicas expresan el fenotipo de una célula $\mathrm{T}$ periférica citotóxica, pudiendo haber reordenamiento clonal del gen de la cadena gamma del TCR.

De forma característica las células neoplásicas no expresan CD3 de superficie, a cambio expresan CD3 intracitoplasmática (cCD3), también denominada CD3 epsilon.

El tratamiento del linfoma NK extraganglionar se basa en la poliquimioterapia. En los estadios localizados se puede intentar el tratamiento combinado con la quimiorradioterpia. Un nuevo estudio se esta llevando a cabo en

\section{TABLA I}

\section{INMUNOFENOTIPO DEL LINFOMA T/NK EXTRAGANGLIONAR}

CD2, CD56, cCD3* ${ }^{*}$ VEB: positivos (fenotipo NK)**

Moléculas-proteínas asociadas a gránulos citotóxicos

(TIA-1, Granzime B, perforina): positivos

CD3, CD4, CD5, CD8: negativos

CD19,CD20,CD22,CD79a (antígenos B): negativos

cCD3: Se caracteriza por el hecho de que el CD3 de superficie es negativo, mientras que el CD3 intracitoplasmático o CD3 epsilon es positivo.

**En algunos casos el CD56 es negativo (CD2+,cCD3+,VEB+), tratándose en estos casos de un linfoma extraganglionar de fenotipo $T$.
Japón con el uso de la quimiorradiotrapia concomitante 5. Sin embargo, en los estadios avanzados el tratamiento se basa en la poliquimioterpia sola. En nuestro caso nos hemos decantado por el esquema de Hyper-CVAD/MTXHIDAC (Tabla II), que se trata de un régimen complejo que incluye dos partes, el segundo de los cuales contiene metotrexate y citarabina a dosis altas, con quimioprofilaxis del sistema nervioso central, seguido de radioterapia. Algunos autores japoneses proponen en los estadios diseminados un régimen de quimioterapia basada en L-asparaginasa (L-asparaginasa, vincristina y prednisona) cada 21 días (13). En todos los pacientes candidatos a trasplante autólogo de médula ósea se debe plantear dicho procedimiento tanto en primera como en segunda línea de tratamiento $(14,15)$. Algunos autores proponen incluso el trasplante alogénico de médula ósea en pacientes seleccionados (16).

\section{TABLA II}

HYPERCVAD/MTX-HIDAC

HyperCVAD: Primera parte del ciclo

Ciclofosfamida* $300 \mathrm{mg} / \mathrm{m}^{2} / 12$ h IV días $1-3$

Doxorrubicina $50 \mathrm{mg} / \mathrm{m}^{2}$ IV día 4

Vincristina $2 \mathrm{mg}$ IV días 4 y $11^{*}$ *

Dexametasona $40 \mathrm{mg} /$ día VO días 1-4 y 11-14

MTX-HIDAC: Segunda parte del ciclo

Metotrexate ** $200 \mathrm{mg} / \mathrm{m}^{2}$ IV en infusión de dos horas día 1 , seguido de $800 \mathrm{mg} / \mathrm{m}^{2}$ IV en infusión continua de 24 horas día 1 Citarabina**** $3 \mathrm{~g} / \mathrm{m}^{2} / 12$ h IV días 2 y 3

Metilprednisolona $50 \mathrm{mg} / 12 \mathrm{~h}$ IV días 1-3

IV: intravenoso; VO: Vía oral; HIDAC: dosis altas de citarabina.

* La ciclofosfamida requiere rescate con Mesna.

* * La vincristina del día 11, sólo se puede administrar si así lo permite la situación clínica y analítica del paciente.

***El metotrexate requiere rescate con ácido folínico.

**** La citarabina requiere ajuste de $\operatorname{dosis}\left(1 \mathrm{~g} / \mathrm{m}^{2} / 12 \mathrm{~h}\right)$ en pacientes mayores de 60 años y en los que la creatinina sérica está por encima de 2 mg.

\section{Bibliografía}

1. Chan JK, Sin VC, Wong KF. Nonnasal lymphoma expressing the natural killer cell marker CD56: a clinicopathologic study of 49 cases of an uncommon aggressive neoplasm. Blood 1997; 89: 4501-05

2. Ferry JA, Sklar J, Zukerberg LR, Harris NL. Nasal lymphoma. A clinicopathologic study with immunophenotypic and genotypic analysis. Am J Surg Pathol 1991; 15: 268-74.

3. Jaffe ES, Chan JK, Su IJ, et al. Report of the Workshop on Nasal and Related Extranodal Angiocentric T/Natural Killer Cell Lymphomas. Definitions, differential diagnosis, and epidemiology. Am J Surg Pathol 1996; 20: 103-10.

4. Cuadra-García I, Proulx GM, Wu CL. Sinonasal lymphoma: a clinicopathologic analysis of 58 cases from the Massachusetts General Hospital. Am J Surg Pathol 1999; 23: 1356-67.

5. Oshimi K. NK cell Lymphoma. Int J Hematol 2002; 76: Suppl 2: 118-21.

6. Ho FC, Srivastava G, Loke SL, et al. Presence of Epstein-Barr virus DNA in nasal lymphomas of B and "T" cell type. Hematol Oncol 1990; 8: 271-80.

7. García-Cosio M, Santon A, Mendez MC, Rivas C, Martin C, Bellas C. Nasopharyngeal /nasal type T/NK lymphomas: analysis of 14 cases and review of the literature. Tumori 2003; 89: 278-84.

8. Porcu P, Baiocchi RA, Magro C. Recent developments in the biology and therapy of T-cell and natural killer-cell lymphomas. Curr Opin Oncol 2003; 15: 353-62.

9. Cuadra-García I, Proulx GM, Wu CL. Sinonasal lymphoma: a clinicopathologic analysis of 58 cases from the Massachusetts General Hospital. Am J Surg Pathol 1999; 23: 1356-62. 
10. Soler J, Bordes R, Ortuno F. Aggressive natural killer cell leukaemia/lymphoma in two patients with lethal midline granuloma. $\mathrm{Br} \mathrm{J}$ Haematol 1994; 86: 659-66.

11. Kwong YL, Chan AC, Liang R.CD56+ NK lymphomas: clinicopathological features and prognosis. Br J Haematol 1997; 97: 821-27.

12. Elenitoba-Johnson KS, Zarate-Osorno A, Meneses A.Cytotoxic granular protein expression, Epstein-Barr virus strain type, and latent membrane protein-1 oncogene deletions in nasal T-lymphocyte/natural killer cell lymphomas from Mexico. Mod Pathol 1998; 11: 754-61.

13. Obama K, Tara M, Niina K. L-asparaginase- based induction therapy for advanced extranodal NK/T-cell Lymphoma. Int J Hematol 2003; 78: 248-50.
14. Liang R, Chen F, Lee CK, et al. Autologous bone marrow transplantation for primary nasal T/NK cell lymphoma. Bone Marrow Transplant 1997; 19: 91-9.

15. Nawa Y, Takenaka K, Shinagawa K, et al. Successful treatment of advanced natural killer cell lymphoma with high-dose chemotherapy and syngeneic peripheral blood stem cell transplantation. Bone Marrow Transplant 1999; 23: 1321-32.

16. Kawa K, Okamura T, Yasui M, Sato E, Inoue M. Allogeneic hematopoietic stem cell transplantation for Epstein-Barr virus associated T/NK-cell lymphoproliferative disease. Crit Rev Oncol Hematol 2002; 44: 251-7. 